Mens

revue d'histoire intellectuelle de l'Amérique française

\title{
L'influence du radicalisme français au Québec au début du XX siècle
}

\section{Éric Leroux}

Volume 6, numéro 2, printemps 2006

URI : https://id.erudit.org/iderudit/1024302ar

DOI : https://doi.org/10.7202/1024302ar

Aller au sommaire du numéro

\section{Éditeur(s)}

Centre de recherche en civilisation canadienne-française

ISSN

1492-8647 (imprimé)

1927-9299 (numérique)

Découvrir la revue

Citer cet article

Leroux, É. (2006). L'influence du radicalisme français au Québec au début du $\mathrm{XX}^{\mathrm{e}}$ siècle. Mens, 6(2), 167-204. https://doi.org/10.7202/1024302ar
Résumé de l'article

Malgré la très grande influence exercée par l'Église catholique sur la société québécoise au tournant du siècle dernier, nous savons aujourd'hui que le pluralisme idéologique existait au Québec et que le radicalisme constituait une des composantes de cette pluralité. Il ne fait pas doute que certains éléments de la société québécoise de l'époque étaient perméables aux idées de gauche en provenance de l'Europe, et plus particulièrement de la France. Ainsi, certaines organisations comme la Ligue de l'Enseignement, l'aile gauche du Parti libéral du Québec dirigée par Godfroy Langlois, les loges maçonniques

L'Émancipation et Force et courage de même que certains membres des syndicats internationaux regroupés au sein du Parti ouvrier étaient influencés par le radicalisme français. Comme cet article le montre, l'alliance qui unira pour un certain temps les libéraux radicaux aux syndicalistes internationaux participait activement de ce courant radical. 


\title{
L'INFLUENCE DU RADICALISME FRANÇAIS AU QUÉBEC AU DÉBUT DU XX SIÈCLE $^{1}$
}

\begin{abstract}
Éric Leroux
École de bibliothéconomie et des sciences de l'information

Université de Montréal
\end{abstract}

\section{Résumé}

Malgré la très grande influence exercée par l'Église catholique sur la société québécoise au tournant du siècle dernier, nous savons aujourd'hui que le pluralisme idéologique existait au Québec et que le radicalisme constituait une des composantes de cette pluralité. Il ne fait pas doute que certains éléments de la société québécoise de l'époque étaient perméables aux idées de gauche en provenance de l'Europe, et plus particulièrement de la France. Ainsi, certaines organisations comme la Ligue de l'Enseignement, l'aile gauche du Parti libéral du Québec dirigée par Godfroy Langlois, les loges maçonniques L'Émancipation et Force et courage de même que certains membres des syndicats internationaux regroupés au sein du Parti ouvrier étaient influencés par le radicalisme français. Comme cet article le montre, l'alliance qui unira pour un certain temps les libéraux radicaux aux syndicalistes internationaux participait activement de ce courant radical.

\section{Abstract}

Though the Roman Catbolic Church exerted a great deal of influence over Quebec society at the beginning of the twentieth century, we are now aware that 
ideological pluralism existed in the province and that radicalism was part of that pluralism. It is indeed evident that certain groups were influenced by left-wing ideas emanating from Europe, and in particular from France. As such, certain organizations, including the Ligue de l'Enseignement, the Liberal party's left wing lead by Godfroy Langlois, the Masonic Lodges L'Émancipation and Force et courage, as well as certain members of the international unions which were united under banner of the Labour Party were influenced by French radicalism. As this paper shows, the temporary alliance between the liberal progressives and the labour leaders was an active part of that radical ideology.

Longtemps, les historiens, les sociologues et les politicologues qui se sont penchés sur le Québec ont prôné la thèse $\mathrm{du}$ monolithisme idéologique pour expliquer la première moitié du $\mathrm{XX}^{\mathrm{e}}$ siècle. À la suite du déclin du libéralisme radical — incarné par les « Rouges" - à la fin du XIX siècle et de la montée de l'ultramontanisme au même moment, le Québec serait entré dans le nouveau siècle sous la dominance d'une idéologie clérico-nationaliste où primaient les valeurs conservatrices d'une Église catholique omnipotente et omniprésente. Le pluralisme idéologique ne se serait finalement manifesté dans le paysage québécois qu'au lendemain de la Deuxième Guerre grâce au "réveil » de l'intelligentsia québécoise. Les thèses de Pierre Elliott Trudeau, Fernand Ouellet, Marcel Rioux, Hubert Guindon, Kenneth McRoberts et Dale Postgate, entre autres, défendent cette vision de l'accession d'un Québec moderne seulement au moment de la Révolution tranquille $^{2}$. Or, depuis l'explosion de la recherche dans le champ des sciences sociales survenue au début des années 1970, les historiens remettent cette interprétation en cause et défendent plutôt l'idée que si l'idéologie clérico-nationaliste exerçait une influence considérable sur la société québécoise, le courant libéral était également présent et constituait un contrepoids avec lequel il fallait compter. En outre, pour certains, la tradition libérale radicale ne semble pas s'éteindre 
avec la seconde condamnation de l'Institut canadien de Montréal (1869), la disparition du Pays (1871), l'exil de Louis-Antoine Dessaulles (1875) ou la mort d'Arthur Buies (1901) ${ }^{3}$.

Or des recherches récentes tendent à démontrer qu'il existerait une parenté idéologique entre le radicalisme français du tournant du $\mathrm{XX}^{\mathrm{e}}$ siècle et certains militants syndicaux et politiques québécois ${ }^{4}$. En effet, il ne fait pas de doute que certaines organisations comme la Ligue de l'enseignement, l'aile gauche du Parti libéral du Québec dirigée par Godfroy Langlois, les loges maçonniques L'Émancipation et Force et courage de même que certains membres des syndicats internationaux regroupés au sein du Parti ouvrier sont influencés par le radicalisme français. Comme nous le verrons, l'alliance qui unira pour un certain temps les libéraux radicaux aux syndicalistes internationaux participe activement de ce courant radical.

\section{La présence du radicalisme français en sol québécois}

Nous entendons par radicalisme, non pas le parti politique, ni les différentes organisations ou factions radicales, mais plutôt un corps de doctrine ou encore une idéologie. En effet, comme le rappelle Gérard Baal :

Le radicalisme du début du siècle, c'est aussi une idéologie, un ensemble de valeurs et de convictions qui imprègnent, à des degrés divers, la vision du monde et le comportement politique des hommes qui s'en réclament. Ces convictions, il est banal de le remarquer, sont moins dictées par un système intellectuel qu'inspirées par une série de traditions, de références à l'histoire de l'idée républicaine et du mouvement républicain ${ }^{5}$. 
Apparu dans le sillage de la Révolution française, le radicalisme français se présente, tout au long du $\mathrm{XIX}^{\mathrm{e}}$ siècle, comme une philosophie politique originale axée sur la promotion des principes de laïcité, de liberté et d'égalité. Regroupés autour de Georges Clémenceau, Léon Bourgeois et Jules Ferry, les radicaux se constituent plus fortement en 1901 avec la création du Parti républicain radical et radical-socialiste (Parti radical). Réformistes, les radicaux réclament depuis le milieu du XIX ${ }^{\mathrm{e}}$ siècle l'extension à tous les citoyens de la propriété par la création de coopératives de production et de consommation ${ }^{6}$. À Montréal, les syndicalistes internationaux s'impliqueront activement dans la mise sur pied de magasins coopératifs à partir du début du siècle et ils seront à la base de la création de la première caisse populaire d'épargne et de crédit en $1908^{7}$.

Loin d'être marginaux, les syndicats internationaux représentent la principale force syndicale au Québec dans la première partie du $\mathrm{XX}^{\mathrm{e}}$ siècle. Les syndicats internationaux sont en fait des syndicats nord-américains affiliés à la puissante centrale américaine, la Fédération américaine du travail (FAT). Fondée en 1886 par le cigarier Samuel Gompers, la FAT regroupe plus d'un demi-million de membres au tournant du siècle. Au Canada, elle compte plus de 700 syndicats regroupés pour la plupart en Ontario et au Québec ${ }^{8}$. Au Québec, au début du $\mathrm{XX}^{\mathrm{e}}$ siècle, les syndicats internationaux recrutent surtout les ouvriers de métiers des secteurs de la construction, de la métallurgie et des transports. Malgré la présence des Chevaliers du travail et des syndicats nationaux au tournant du siècle, le syndicalisme international demeure, dans la première moitié du $\mathrm{XX}^{\mathrm{e}}$ siècle, le principal représentant des travailleurs syndiqués au Québec. En effet, il apparait clairement que les syndicats internationaux regroupent les deux tiers 
des syndicats québécois jusqu'aux années 1930, et plus de $40 \%$ dans les années 1940 et 1950 .

Très tôt, le mouvement syndical se dote de structures afin d'améliorer la représentation de ses membres auprès des pouvoirs publics. Fondé en 1886, le Congrès des métiers et du travail du Canada (CMTC) représente les syndicats internationaux sur la scène politique canadienne. Il a pour but de recueillir les doléances de ces syndicats et de les présenter au gouvernement fédéral. L'objectif est de sensibiliser le gouvernement aux problèmes des travailleurs afin qu'il adopte des lois qui leur seront favorables. À Montréal, le Conseil des métiers et du travail (CMTM) peut être considéré comme le pendant municipal du CMTC. Regroupant les sections locales des syndicats internationaux de la région montréalaise, le CMTM regroupent 105 syndicats et 55000 membres en $1919^{\circ}$. Fondé en 1899, le Parti ouvrier est le bras politique des syndicalistes internationaux au Québec. S'inspirant du Parti travailliste anglais, les partisans du Parti ouvrier considèrent que les partis traditionnels sont interchangeables et qu'il faut créer une troisième voie, la seule voie possible en fait pour améliorer les conditions de vie de la classe ouvrière. Ils se démarquent ainsi de la Fédération américaine du travail qui est beaucoup plus tiède à l'endroit de l'action politique ouvrière, favorisant plutôt une action de représentation (lobbying) auprès des gouvernements. Pour les travaillistes québécois, l'action politique est indispensable à la réussite de l'action syndicale et ne peut se définir que par l'existence et le dynamisme d'un parti dirigé par les ouvriers et s'adressant à eux. Pragmatiques, les travaillistes repoussent les socialistes et n'hésitent pas à s'associer aux libéraux radicaux comme nous le verrons plus loin ${ }^{10}$. Malgré plusieurs tentatives pour faire élire des candidats, le Parti ouvrier connaîtra peu de succès, mis à part l'élection d'Alphonse Verville dans la circonscription de Mai- 
sonneuve en février 1906. Réélu à plusieurs reprises, Verville siégera à la Chambre des communes jusqu'en $1921^{11}$.

Héritier des idéaux du siècle des Lumières, le radicalisme français s'articule autour d'idées phares comme la valorisation du progrès (la foi en la science), l'exaltation de l'instruction laïque, gratuite et obligatoire, la valorisation du républicanisme et des principes de liberté (liberté du suffrage, liberté de la presse, liberté de conscience, liberté de réunion et d'association) ${ }^{12}$. En opposition au monarchisme et au cléricalisme, le radicalisme s'inspire de la Révolution française et recrute plusieurs de ses membres au sein de la franc-maçonnerie. Dans un article rédigé en 1927 et intitulé « Hommage à la France ", le dirigeant syndical d'origine belge Gustave Francq traite de l'importance de la Révolution française pour les ouvriers :

Nous savons nous, les ouvriers internationaux, que c'est la Révolution française qui changea la face du monde et fit régner sur la terre tout ce qui est compris dans ces trois grands mots : Liberté, Égalité, Fraternité.

Du reste, n'en déplaise à nos réactionnaires, le Quatorze Juillet est la fête de tous les hommes libres de la terre. Elle est bien un peu notre fête à nous, travailleurs organisés, qui avons senti le grand souffle de la Révolution française et avons également eu bien des Bastilles à renverser. C'est pourquoi nous sommes de tout cœur avec nos compatriotes de France ${ }^{13}$.

Plusieurs syndicalistes liés aux syndicats internationaux et au Parti ouvrier connaissaient l'existence du radicalisme français. Intellectuel du mouvement ouvrier organisé, le dirigeant syndical Gustave Francq occupe des postes d'importance dans les rangs des syndicats internationaux durant une cinquantaine d'années. Fondateur de plusieurs journaux ouvriers, il rédige plus de mille éditoriaux dans les pages du Monde ouvrier 
entre 1916 et 1952. Figure de proue du Parti ouvrier de Montréal entre 1906 et 1916, il oriente les politiques du parti selon les principes travaillistes et présente un projet de société axé sur une plus grande intervention de l'État dans le champ des politiques sociales. Au tournant du siècle, Francq milite dans la franc-maçonnerie où il promeut la séparation de l'Église et de l'État, lutte en faveur d'une réforme en profondeur du système scolaire québécois (obligation, gratuité et laïcité), de l'instauration de coopératives de consommation et de production et de la nationalisation des entreprises de services publics qui exercent un monopole comme la Montreal Street Railway en charge des tramways, la Montreal Light, Heat and Power qui contrôle la distribution d'électricité et la Montreal Water and Power qui détient le monopole sur la distribution de l'eau à Montréal. Or nous savons que Francq connaissait l'existence du radicalisme français et qu'il a sans doute été influencé par ce mouvement idéologique. Dans Le Monde ouvrier, plusieurs articles rédigés par Francq ou des collaborateurs y font référence directement ou indirectement. En 1923, il écrit d'ailleurs à ce sujet :

[...] n'est-il pas vrai que, dans la plupart des pays d'Europe, il y avait tout comme ici, un parti libéral et un parti conservateur ; que dans le groupe libéral il y avait aussi un clan de doctrinaires et un autre clan de progressistes, que le fossé entre les deux s'est creusé de plus en plus [...] et qu'un beau jour les doctrinaires se sont aperçus qu'il y avait plus de communauté d'idées - sinon de principes - entre eux et les conservateurs qu'il y en avait avec les libéraux-progressistes ; ceux-ci, à leur tour, ont découvert qu'il y avait sur beaucoup de points communion d'idées avec le groupe radical [...] et ce qui devait arriver s'est logiquement accompli : le groupe doctrinaire s'est rallié au parti conservateur et le groupe progressiste s'est amalgamé au parti radical ; 
de ce croisement sont nées la Droite réactionnaire et la Gauche radicale ${ }^{14}$.

De même, au tournant du siècle, plusieurs syndicalistes québécois semblent connaître ce courant de pensée. En octobre 1904, par exemple, une délégation française, sous la direction d'Albert Métin, se rend à l'exposition de la ville de Saint-Louis aux États-Unis. Du 12 au 15 octobre, la délégation visite le Québec, principalement les villes de Québec et de Montréal. Des dirigeants ouvriers importants comme Alphonse Verville, député ouvrier de la circonscription de Maisonneuve de 1906 à 1921, Joseph-Alphonse Rodier, chroniqueur ouvrier à La Presse et à La Patrie et principal instigateur de la fondation du Parti ouvrier en 1899, Joseph Ainey, Isidore Tremblay, Narcisse Arcand, et quelques autres sont de la partie. Gustave Francq est également présent puisqu'il accompagne Claude Gignoux, directeur de l'imprimerie coopérative «La Laborieuse » de Nîmes. Dans le compte rendu qu'il fait de sa visite, Métin souligne le fait que les syndicalistes d'ici connaissent le radicalisme français ${ }^{15}$.

En fait, il existe une véritable communauté d'idées entre les réformes sociales défendues par les syndicalistes internationaux au tournant du siècle et les mesures comprises dans le programme de Belleville de 1869 et dans la première plateforme politique du Parti radical en 1907. La similitude est impressionnante. En effet, les principales réclamations des radicaux portent sur la réforme du système électoral (suffrage universel) ; la nationalisation de certains monopoles et des entreprises de services publics ; la démocratisation et la laïcisation de l'éducation; la formation de syndicats et d'associations coopératives par lesquels le travailleur peut faire valoir ses droits et défendre ses intérêts ; le droit pour l'État d'intervenir dans les relations de travail. Enfin, le programme des radicaux prévoit la mise en place d'un vaste programme de 
mesures sociales propres à protéger les travailleurs et les travailleuses : pensions de vieillesse, lois ouvrières sur l'emploi des femmes et des enfants dans l'industrie, sur les accidents de travail, sur la diminution des heures de travail, etc. ${ }^{16}$ Or toutes ces mesures sont également défendues par les syndicalistes internationaux qui se regroupent au sein d'organismes comme le Conseil des métiers et du travail de Montréal (CMTM), le Congrès des métiers et du travail du Canada (CMTC) et le Parti ouvrier ${ }^{17}$.

Autre élément important, tout comme les syndicalistes internationaux, les radicaux s'opposent aux socialistes qui veulent s'approprier l'ensemble des moyens de production. À ce sujet, le programme de 1907 précise : «Le Parti Radical et Radical-Socialiste est résolument attaché au principe de la propriété individuelle dont il ne veut ni commencer ni même préparer la suppression ${ }^{18}$. " Les radicaux rejettent les principes socialistes ainsi que les conceptions de l'école libérale, recherchant plutôt à atteindre un équilibre social afin de poursuivre l'œuvre amorcée par les réformateurs sociaux de la Troisième République. Les "internationaux », qui s'opposent eux aussi à la doctrine socialiste et au libéralisme économique, défendent une vision de la société similaire à celle des radicaux, c'est-à-dire axée sur une conception plus interventionniste de l'État dans le champ des relations de travail et des politiques sociales, sur une meilleure distribution de la richesse (principe d'égalité) sans remettre en cause le principe de la propriété individuelle et du système capitaliste. D'ailleurs, au Parti ouvrier, les travaillistes s'opposent aux socialistes d'Albert Saint-Martin et aux sociaux-démocrates qu'ils expulsent des rangs du Parti ouvrier en $1907^{19}$. Réformistes, les travaillistes ne veulent pas renverser le système capitaliste, mais ils tentent plutôt de le bonifier afin de le rendre acceptable aux yeux de la classe ouvrière. Joseph-Alphonse Rodier écrivait à 
ce sujet en 1906 dans La Patrie : "Le Parti ouvrier et les unions ouvrières forment un groupe tampon entre le capitalisme et la bourgeoisie d'un côté et le socialisme de l'autre ${ }^{20}$. »

Loin de se limiter aux organisations syndicales, le radicalisme se retrouve aussi dans plusieurs sphères de la société québécoise. Directeur et rédacteur en chef de La Patrie de 1895 à 1903, puis du Canada de 1903 à 1910, Godfroy Langlois représente la tendance radicale du Parti libéral. Personnage influent de la scène politique québécoise, il est un ami personnel de Gustave Francq qui a travaillé comme typographe pour les deux journaux officiels des libéraux. Or, Langlois fut influencé par la théorie du solidarisme de Léon Bourgeois, un des penseurs influents du radicalisme français. Selon son biographe, Langlois se met à adopter les principes de Bourgeois dans ses éditoriaux dans le journal Le Canada à partir de 1905. Il n'est pas seul à agir ainsi puisque dès la fin du $\mathrm{XIX}^{\mathrm{e}}$ siècle, le journal Le Signal reconnaissait les grands principes du radicalisme mis de l'avant par Bourgeois : "Dès août 1897, Le Signal, le journal radical des libéraux montréalais, exprime clairement l'admiration que porte à Bourgeois la nouvelle génération de rouges radicaux ${ }^{21}$. ”

Langlois est aussi à la base de la mise sur pied de la Ligue de l'Enseignement en 1902. Fondée par une centaine de personnes le 9 octobre 1902, la Ligue vise surtout à élargir le rôle de l'État en matière d'éducation et fait la promotion de l'obligation scolaire, de l'uniformisation des commissions scolaires à Montréal et de la gratuité et de l'uniformité des livres. Elle s'inspire d'ailleurs de la Ligue française de l'Enseignement présidée par le franc-maçon Ferdinand Buisson et qui a soutenu activement la politique républicaine et les réformes scolaires de Jules Ferry dans les années 1880. À la fondation de la Ligue, Godfroy Langlois occupe le poste de premier vice-président de l'organisme et Paul G. Martineau 
celui de conseiller. Rassemblement de membres de la petite bourgeoisie francophone libérale, les ouvriers sont absents de cette organisation; seule exception, le militant socialiste Albert Saint-Martin, dont le nom apparaît à titre de membre, mais qui ne participera pas aux activités de la Ligue ${ }^{22}$.

Marginalisé au sein du Parti libéral à cause de ses positions radicales et de ses liens avec la loge L'Émancipation dont il est un des piliers, Langlois quitte la direction du $\mathrm{Ca}$ nada en 1910 pour fonder un nouveau journal, Le Pays ${ }^{23}$. Défendant des idées similaires, comme la réforme du système scolaire et des mœurs électorales et la lutte contre les compagnies à monopole (électricité, gaz, transport en commun), Francq et Langlois font alliance pour combattre des ennemis communs - principalement l'Église, le gouvernement libéral et les nationalistes - et ils militent ensemble au sein de la franc-maçonnerie et au journal Le Pays jusqu'à la Première Guerre mondiale. Ce sont, par exemple, les pressions conjointes des syndicalistes internationaux et des libéraux radicaux qui mènent à la création de la Commission royale d'enquête sur l'éducation de 1909 comme nous le verrons dans la deuxième partie de ce texte. En 1914, Langlois quitte définitivement le Québec pour s'installer en Belgique à titre de représentant commercial du Québec. Il meurt à Bruxelles le 6 avril 1928 à l'âge de 61 ans.

\section{La franc-maçonnerie québécoise}

En France, l'anticléricalisme du mouvement radical le rapproche instinctivement de la franc-maçonnerie. Comme le souligne Gérard Baal, ce n'est pas un hasard si le président du Grand Orient de France (GODF) est remplacé en 1885 par un radical ${ }^{24}$. Or, même si dans le Québec fortement catholique du tournant du siècle la situation est différente, il y a lieu de croire que certains francs-maçons de L'Émancipation et 
de Force et courage s'inspiraient largement du radicalisme français. Fondée en 1896 par des francs-maçons francophones qui désirent s'affranchir de la tutelle anglaise de la Grand Lodge of Quebec, la loge L'Émancipation est rattachée à la contrepartie française de la Grande Loge Unie d'Angleterre, soit le Grand Orient de France (GODF). Contrairement à la maçonnerie britannique qui accepte et promeut l'existence de Dieu - le Grand Architecte de l'Univers -, la maçonnerie française, par le biais du GODF, propose un modèle plus libre de croyance axé plutôt sur les idéaux de la Révolution française et la valorisation de la séparation de l'Église et de l'État. Ainsi, selon Paul Nadon, "la franc-maçonnerie n'est ni déiste, ni athée, ni même positiviste. Institution pratiquant et affirmant la solidarité humaine, elle est étrangère à tout dogme et à tout crédo religieux. Elle a pour principe unique le respect absolu de la liberté de conscience ${ }^{25}$ \%. Imprégné des idéaux de la Troisième République, L'Émancipation, qui prend pour devise "Raison, Travail, Liberté » au moment de sa création, propose une vision de la société axée essentiellement sur l'idéal de la liberté :

Il est formé à Montréal, Canada, une société d'hommes probes qui, liés par des sentiments de liberté, d'égalité, de fraternité, travaillent individuellement et en commun à la réalisation des progrès sociaux, exerçant ainsi la bienfaisance dans le sens le plus étendu. — Le but principal qu'ils poursuivent, c'est l'affranchissement intellectuel du peuple canadien, encore courbé sous le despotisme clérical, en créant une vérité morale et libre $^{26}$.

Influencée par les lois françaises sur l'éducation de Jules Ferry, la franc-maçonnerie québécoise met en tête de son programme la démocratisation de l'éducation fondée sur trois principes: la gratuité, l'obligation et la laïcisation. C'est pourquoi au début du siècle, les maçons de L'Émancipation, Godfroy Lan- 
glois en tête, participeront aux activités de la Ligue de l'enseignement. Pour les maçons, l'enseignement est la pierre d'assise de leur projet de société axé sur la valorisation des libertés fondamentales par " l'affranchissement intellectuel du peuple canadien encore courbé sous le despotisme clérical ", comme le mentionne la constitution de L'Émancipation ${ }^{27}$. Outre la Ligue de l'enseignement, les maçons tenteront de faire passer leur message à travers des cercles d'étude comme le cercle Alpha-Oméga et grâce à certains journaux comme Les Débats, La Petite Revue ou Le Pays.

Si la loge l'Émancipation est principalement composée de membres des professions libérales, il en est tout autrement de Force et courage dont les ouvriers comptent pour près de la moitié des effectifs ${ }^{28}$. Pour le docteur Alfred Marcil, qui met sur pied Force et courage en janvier 1910, la réforme de la société doit venir de la classe ouvrière et non de la petite bourgeoisie libérale comme le préconisait les membres de L'Émancipation. Ainsi tout en poursuivant les objectifs de L'Émancipation en ce qui a trait à la réforme de l'éducation et tout en demeurant attaché au Grand Orient de France, certains membres de la nouvelle loge comme Gustave Francq, Édouard Henry, lui aussi belge d'origine, membre du syndicat international des lambrisseurs de navires et agent d'affaires dans le port de Montréal, et Fernand Marrié, journaliste au Pays et au Monde ouvrier, amènent la loge à s'occuper de questions sociales plus larges: réformes scolaires élargies avec l'ajout de l'uniformité et de la gratuité des manuels scolaires et l'amélioration des conditions de travail des instituteurs et institutrices; réformes municipales visant l'amélioration des conditions de vie de la classe ouvrière, lutte contre la hausse constante du coût de la vie dans les années 1910 et valorisation du mouvement coopératif, amélioration du statut juridique de la femme par la reconnaissance du droit de vote, etc. 
C'est principalement par la voix du journal Le Pays que ces maçons font passer leur message. À l'automne 1913, par exemple, le journal organise une campagne contre les logements insalubres et la mortalité infantile à Montréal et il réclame la diminution du coût de la vie. En 1917, tout comme Le Monde ouvrier, Le Pays se prononce vigoureusement contre la prohibition.

Il existe une similarité entre les idéaux défendus par plusieurs dirigeants syndicaux internationaux et ceux de Force et courage. Le dirigeant syndical Gustave Francq, par exemple, accorde beaucoup d'attention à défendre certaines idées comme la liberté d'expression, la valorisation des institutions démocratiques jumelée au perfectionnement du processus électoral (grâce à des mesures comme le suffrage universel, le droit de vote des femmes, l'abolition de la qualification foncière, la représentation proportionnelle et même le droit de vote obligatoire), l'égalité des chances pour tous par une meilleure répartition de la richesse et, évidemment, la réforme du système scolaire québécois. Il apparait clairement qu'il existe une corrélation entre les principes défendus par Force et courage et ceux défendus par nombre de syndicalistes internationaux comme Francq. D'ailleurs, à l'instar des syndicalistes internationaux, Francq ne se fait pas prier pour défendre un autre grand principe des francs-maçons et des radicaux français : l'anticléricalisme. Il s'oppose en effet radicalement à l'intervention du clergé dans la vie publique. Ses principales interventions sur le sujet se produiront à la fin des années 1910 au moment de l'avènement des syndicats catholiques qui profitent largement de l'appui de l'Église. Au credo des militants catholiques "Hors de l'Église, point de salut !", Francq répond : "S'il est vrai que les ouvriers catholiques sont tenus de croire à l'infaillibilité des dogmes de l'Église, il est tout aussi vrai que cela ne s'applique qu'aux questions spirituelles. 
[...] Nous l'avons déjà dit et nous ne cesserons de le répéter: si le clergé veut garder le respect et la confiance que le peuple a pour lui, qu'il reste en dehors de luttes politiques et économiques ou des questions purement matérielles ${ }^{29}$. " À ses yeux, vouloir combiner les intérêts matériels aux valeurs spirituelles ne peut conduire qu'à l'échec "car tôt ou tard, l'un des deux intérêts prendra le dessus ${ }^{30}$ ». Francq n'est pas le seul à penser ainsi. Le mémoire du Parti ouvrier présenté à la Commission royale d'enquête sur l'éducation de 1909-1910 précise que la question des réformes scolaires "n'est pas une question religieuse, mais uniquement nationale $»$. Enfin, au tournant des années 1920, les «internationaux » combattent vigoureusement l'implantation des syndicats catholiques dans les industries québécoises ${ }^{31}$.

La franc-maçonnerie est le point de rencontre des membres des professions libérales, de gens issus des milieux d'affaires, de politiciens, d'artistes, de journalistes et de syndicalistes. En ce qui a trait à l'allégeance politique de ses membres, nul doute que la plupart sont de francs libéraux issus du courant radical. Parmi les libéraux radicaux, on y rencontre le journaliste et avocat Gonzalve Désaulniers, l'artiste-peintre Ludger Larose, les frères Adelstan et Paul Le Moyne De Martigny, respectivement chirurgien et journaliste, les journalistes Arthur Beauchesne et Arsène Bessette et le dentiste Gaston Maillet. De plus, s'il est certain que Francq côtoie Godfrois Langlois à la loge L'Émancipation de 1908 à 1910, peut-être y a-t-il aussi rencontré le fougueux député libéral de SaintHyacinthe, T.-D. Bouchard.

Si, pour sa part, elle n'est pas membre de la franc-maçonnerie - seuls les hommes peuvent y adhérer —, la directrice de la première bibliothèque municipale de Montréal et journaliste Èva Circé-Côté est associée de très près aux milieux francs-maçons. En effet, le docteur Pierre-Salomon Côté 
qu'elle épouse en 1905 était reconnu pour ses sympathies franc-maçonnes. Lorsqu'il décède en décembre 1909, plus de 400 parents et amis assistent aux funérailles civiles du docteur Côté, un événement peu commun à l'époque qui provoquera une certaine indignation dans la population et dans la presse montréalaise ${ }^{32}$. Avant son arrivée au Monde ouvrier en 1916, elle participe à la publication de journaux comme Les Débats ou Le Pays dont l'allégeance à la franc-maçonnerie n'est plus à démontrer. Sous la plume de Julien Saint-Michel, elle connaitra une longue carrière au Monde ouvrier publiant jusqu'à une cinquantaine d'articles par années. La modernité, l'éducation, la démocratie et le féminisme sont les principaux thèmes que Circé-Côté aborde durant ses vingt-deux ans de collaboration avec le journal de Francq. Selon sa biographe, Andrée Lévesque : " [...] elle affiche des sympathies socialistes et des opinions anticléricales, elle réclame l'intervention de l'État dans les services sociaux, elle défend le cinéma et cite Rousseau, Voltaire, Hugo et Zola, tous à l'Index de l'Église catholique. Enfin, elle fait l'éloge de l'industrie et du progrès ${ }^{33} \%$.

\section{L'alliance des syndicalistes internationaux et des libéraux radicaux}

Durant la période 1896-1914, il existe une importante conjonction d'intérêts entre les syndicalistes internationaux et l'aile gauche du Parti libéral du Québec. Une conjonction d'intérêts qui se manifestera en définitive par la formation d'alliances stratégiques dans le but de promouvoir certaines mesures sociales particulières. Or, l'idéologie radicale semble véritablement inspirer la réunion de ces deux groupes; d'ailleurs, la question de la réforme scolaire et celle de la démocratisation des institutions politiques québécoises demeurent la pierre d'assise de cette alliance. 
Dans le paysage idéologique québécois du tournant du siècle, il ne fait pas de doute que les ouvriers sont beaucoup plus près du libéralisme que du conservatisme. Dans l'ensemble canadien, les ouvriers, et surtout les dirigeants ouvriers, font majoritairement partie de la base électorale du Parti libéral. Par exemple, les trois députés ouvriers élus au Parlement fédéral au tournant du siècle, Arthur Puttee, éditeur du journal The Voice de Winnipeg, Ralph Smith de Nanaimo, Vancouver, et Alphonse Verville, se retrouveront assez rapidement dans le camp des libéraux. À Montréal, J.-A. Rodier, membre fondateur du Parti ouvrier en 1899, a été chroniqueur ouvrier aux deux grands journaux libéraux de la métropole: La Presse et La Patrie.

Les ouvriers croyaient que l'arrivée au pouvoir des libéraux de Laurier à Ottawa en 1896 et de Félix-Gabriel Marchand l'année suivante à Québec marquerait l'adoption de réformes sociales majeures, entre autres, dans le champ de l'éducation et concernant la question de la qualification foncière à Montréal. Au cours de sa campagne électorale, F.-G. Marchand promettait, par exemple, d'instaurer la gratuité des livres scolaires s'il était élu, une revendication majeure des ouvriers au tournant du siècle ${ }^{34}$.

Les ouvriers ne sont pas les seuls à être déçus du peu d'empressement du gouvernement libéral à remplir ses promesses. Au sein du Parti libéral, un groupe de libéraux radicaux, mené par le député de Montréal-Saint-Louis, Godfroy Langlois, ne cesseront de faire pression sur le gouvernement libéral de Marchand, de Simon-Napoléon Parent et de Lomer Gouin à partir de 1905. Selon Patrice Dutil, le biographe de Langlois, les radicaux sont des "vétérans libéraux dégoûtés des inclinations conservatrices de leurs chefs ${ }^{35} »$. De vieux "Rouges» donc qui tentent d'adapter les idées radicales du $\mathrm{XIX}^{\mathrm{c}}$ siècle aux nouvelles réalités engendrées par l'urbanisa- 
tion, l'industrialisation et la montée du mouvement ouvrier organisé. Ainsi, selon Dutil :

Leur objectif est de rendre vivante l'idéologie du libéralisme pour qu'elle réponde de façon pratique aux changements qui bouleversent le monde et assure la survie du système bourgeois capitaliste ainsi que la préservation des principes de libertés individuelles et d'économie de marché. Pour les libéraux progressistes, cela implique la remise en question d'un libéralisme voué à la "liberté » économique et l'imposition d'un nouveau libéralisme qui profite à la majorité, plutôt qu'à une minorité, de la population ${ }^{36}$.

Si Langlois doit être considéré comme le chef de la faction radicale, il faut mentionner qu'il n'est pas seul à se réclamer de ce "nouveau » courant idéologique que Dutil qualifie de libéralisme "progressiste ». Au sein du Parti libéral, les radicaux forment une aile bien distincte, l'aile gauche du parti. Durant une dizaine d'années, entre 1897 et 1908, une douzaine de libéraux adhèreront à un moment ou à un autre au libéralisme radical. L'historien Patrice Dutil laisse même entendre que l'aile radicale du Parti libéral aurait été assez puissante pour réussir à renverser le régime de Simon-Napoléon Parent en mars 1905 et le remplacer par Lomer Gouin comme nouveau premier ministre ${ }^{37}$. Le torchon brûlait entre Parent et les radicaux de Langlois depuis 1901, année où Parent avait contribué à faire battre le projet de loi du député libéral de Shefford, Tancrède Boucher de Grosbois, en faveur de l'obligation scolaire et où il avait cautionné le trust de la Montreal Light, Heat and Power Company (MLHPC) en lui octroyant une charte pour l'exploitation de l'électricité et du gaz à Montréal ${ }^{38}$.

Sur la scène municipale montréalaise, les appuis au libéralisme radical sont tout aussi importants. À l'élection mu- 
nicipale de 1904, on atteint un sommet avec l'élection ou la réélection de dix-neuf échevins qui adhèrent à ce courant. Ses principaux défenseurs sont : Camille Piché, L.-A. Lapointe, George Sadler, Daniel Gallery, Arsène Lavallée, Henry Ekers, futur maire de Montréal de 1906 à 1908, S. D. Vallières et Paul G. Martineau qui est à l'origine de la première bibliothèque municipale fondée en novembre 1904 et qui s'adresse spécifiquement à la classe ouvrière ${ }^{39}$. Selon l'historien Robert Gagnon, Martineau, qui est membre de la Ligue de l'Enseignement, comme nous l'avons vu plus tôt, et sympathisant de la loge maçonnique l'Émancipation, milite en faveur de l'instauration de mesures progressistes à la Commission des écoles catholiques de Montréal (CÉCM) et doit être considéré comme une figure importante de la scène municipale montréalaise du début du siècle ${ }^{40}$. Ancien rédacteur du journal radical Le Signal, Martineau n'est pas un nouveau venu sur la scène politique montréalaise, lui qui réclame depuis 1882 l'école gratuite et obligatoire ${ }^{41}$. Très près des organisations syndicales de la métropole, Martineau est décrit dans le programme de la Fête du travail de 1900 comme l'ami sincère des ouvriers, celui dont le nom est devenu " une véritable puissance parmi les classes ouvrières ${ }^{42} »$. Enfin, le libéralisme radical du tournant du siècle peut compter aussi sur les milieux littéraires et journalistiques montréalais ${ }^{43}$.

Sur la scène politique québécoise, la question de l'éducation revient comme un leitmotiv depuis le milieu du $\mathrm{XIX}^{\mathrm{e}}$ siècle et plus particulièrement depuis l'abolition du ministère de l'Éducation en 1875. En décembre 1897, par exemple, le gouvernement libéral de F.-G. Marchand présente un projet de loi en faveur de la création d'un ministère de l'Éducation en remplacement du surintendant de l'Instruction publique, ce qui aurait pour conséquence de diminuer l'influence de l'Église, mais le projet de loi sera finalement battu par les 
conservateurs qui détiennent la majorité au Conseil législatif. Par contre, il faut aussi mentionner que depuis le tournant du siècle, l'État a commencé à légiférer dans le secteur de l'éducation. La création des écoles du soir par Honoré Mercier, de même que la création de l'école des Hautes études commerciales (HÉC) et la mise sur pied d'écoles techniques et commerciales par Lomer Gouin en constituent de bons exemples ${ }^{44}$.

La démocratisation de l'éducation est au cœur des réclamations des syndicalistes internationaux et des libéraux radicaux depuis la fin du XIX ${ }^{e}$ siècle. Au Québec, les syndicalistes internationaux réclament l'instruction gratuite et obligatoire depuis 1892, l'uniformité des manuels scolaires à partir de 1902 et la création d'un ministère de l'Instruction publique depuis 1904. En 1906, le Conseil des métiers et du travail de Montréal (CMTM), qui chapeaute les syndicats internationaux à Montréal, radicalise ses positions et présente un réquisitoire en faveur de la laïcisation des écoles et d'une augmentation des salaires des instituteurs et institutrices laïques que l'on traite "sans justice ». On réclame aussi d'autres mesures depuis le tournant du siècle, comme la création de bibliothèques publiques, la gratuité des écoles du soir, la création d'écoles techniques et commerciales et la centralisation des commissions scolaires à Montréal ${ }^{45}$.

Chez les syndicalistes internationaux, le dirigeant Gustave Francq est certes l'un des plus ardents défenseurs de la réforme de l'éducation avec les Alphonse Verville, Narcisse Arcand, Zotique Lespérance, Joseph Ainey, Achille Latreille, G.-R. Brunet et quelques autres. Jusqu'à la Première Guerre mondiale, plus de la moitié des interventions de Francq aux congrès annuels du Congrès des métiers et du travail du Canada (CMTC) porte sur des questions touchant l'éducation. Robert Rumilly, qui met l'accent dans sa présentation de Francq sur ses origines belges, dresse un juste portrait du per- 
sonnage lorsqu'il mentionne : "Gustave Francq croyait aux vertus libératrices de l'instruction. Il opposait la Lumière et l'Obscurantisme. Il répétait une devise des vieux syndiqués européens - une vraie formule à la Victor Hugo : "Un peuple est fort quand il sait lire." Or, ce militant, ce convaincu, exerçait une influence prépondérante au Conseil des Métiers et du Travail de Montréal. [...] Stimulés par Gustave Francq, les syndicats réclamaient toujours le rétablissement du ministère de l'Instruction publique ${ }^{46}$. " Confrontés à l'immense pouvoir de l'Église catholique qui cherche à garder la mainmise sur le système scolaire québécois et s'oppose à toute intervention de l'État, les syndicalistes internationaux luttent ferme pour tenter d'influencer les pouvoirs publics.

De son côté, Langlois exige des réformes dans le système scolaire québécois depuis les années 1890. Influencé par les réformes scolaires qui ont lieu en France à la même époque - les lois Combes - la mise en place d'un système public d'enseignement obligatoire, libre et non confessionnel est au cœur de son programme réformiste ${ }^{47}$. Libre penseur et digne successeur des "Rouges » du XIX ${ }^{e}$ siècle, Langlois est donc un farouche partisan du contrôle de l'État sur l'éducation et de la laïcisation de l'enseignement : "L'enseignement laïc! Encore une machine diabolique en faveur de laquelle nous nous prononçons hautement parce qu'elle est dans l'intérêt populaire et parce que l'État doit faire tous ses efforts pour instruire et éclairer ceux qui paraissent avoir été systématiquement plongés dans l'ignorance ${ }^{48}$. " À partir de la fin des années 1890, il abordera aussi la question de l'éducation dans une perspective ouvrière.

Habitué de fréquenter des politiciens qui n'osent pas se prononcer de façon claire et précise sur des questions touchant le pouvoir du clergé, Langlois est fier de pouvoir compter sur des alliés qui n'ont pas peur de réclamer haut et fort 
des réformes sociales majeures. C'est du moins ce qu'il écrit au député ouvrier, Alphonse Verville, en février 1912:

J'ai une grande admiration pour vous et pour vos camarades les chefs ouvriers Ainey, Francq, Giroux, Arcand et les autres, parce que vous faites de belles luttes désintéressées pour les idées. Vous êtes seuls à marcher à l'avant-garde dans ce pays où l'hypocrisie est de rigueur. Sans crainte des coups qui pleuvent, sans fausse honte, vous faites la bataille du progrès, vous réclamez des réformes, et cela seulement est énorme chez nous qui avons une horreur instinctive de tout ce qui n'est pas routine et ornière ${ }^{49}$.

Langlois n'est pas seul dans le camp des libéraux à réclamer la laïcisation du système d'enseignement. À Ottawa, le député fédéral de Saint-Jacques, Honoré Gervais, réclamait depuis quelques années la création d'une école des HÉC basée sur le modèle européen, tandis qu'à Montréal, le projet de laïcisation des écoles est soutenu par l'échevin Paul G. Martineau et par le CMTM. Dans son Histoire de Montréal, Rumilly se questionne sur la place du clergé face à un tel projet: «Les écoles souhaitées par Gervais, par Langlois, par Ainey, par Francq, seraient des écoles d'État, donc laïques. Que faire de religieux dans l'enseignement commercial et technique ${ }^{50}$ ?"

\section{La Commission royale d'enquête sur l'éducation (1909-1910)}

Au début du siècle, à Montréal, le débat le plus important porte sur la question de la centralisation des commissions scolaires. Le nombre élevé de commissions scolaires plus d'une trentaine - constitue un problème majeur pour les parents puisque ces commissions jouissent d'une entière liberté en ce qui a trait à la sélection des livres apparaissant au programme. Ainsi, les livres utilisés variant d'une commis- 
sion scolaire à l'autre, chaque déménagement entraîne des coûts considérables pour les familles ouvrières qui doivent renouveler le matériel pédagogique de leurs enfants ${ }^{51}$. Dans ces circonstances, le nombre élevé de commissions scolaires à Montréal est préjudiciable aux intérêts des travailleurs. Leur fusion permettrait d'obtenir beaucoup plus facilement l'uniformité des livres et une diminution des coûts liés à l'éducation.

Dans ce but, le CMTM, le Comité exécutif de la province de Québec (CEPQ) et le Parti ouvrier ne cessent de faire pression sur le gouvernement provincial et sur les échevins de la ville en faveur de l'uniformité des manuels scolaires. En septembre 1909, par exemple, Gustave Francq présente au congrès du CMTC une résolution favorisant l'uniformité et la gratuité des manuels scolaires pour l'ensemble des écoliers de la province. Selon lui, les deux tiers des livres sont imprimés par les communautés religieuses qui les vendent au plus haut prix possible, tout en les gardant au programme le moins longtemps possible ${ }^{52}$. Appuyé par Joseph Ainey et par Alphonse Verville, deux piliers du syndicalisme international au Québec, il fait une sortie en règle contre le gouvernement québécois qui ne rend pas justice à la classe ouvrière et contribue à la maintenir dans un état d'infériorité en cautionnant l'action des communautés religieuses. En agissant ainsi, Francq ne choque pas uniquement les membres du gouvernement, mais aussi les autorités religieuses. En effet, pour ces dernières, la gratuité apparaît comme un prélude à l'école neutre et laïque, tandis que l'uniformité des livres pourrait aboutir éventuellement à la mainmise de l'État sur leur contenu.

Dans L'Action sociale, l'éditorialiste Jules Dorion s'en prend aux chefs ouvriers, leur conseillant de ne pas se mêler de sujets qu'ils ne connaissent pas. Selon Dorion, l'uniformité des manuels scolaires signifie que les enfants de diffé- 
rentes religions - catholiques, protestants et juifs - devront tous utiliser les mêmes livres : "Ce serait, à brève échéance, le triomphe de la médiocrité, l'égalité banale et terre à terre, et peut-être la perte de tout sens moral $^{53}$ ». Dans ce même éditorial, Dorion ne se gêne pas pour qualifier Francq d'athée, d'ennemi de la doctrine catholique et de disciple de la révolution française. La même année, une série d'assemblées publiques organisée par le Parti ouvrier indispose certains journaux. À Québec, le journal catholique ultramontain, La Vérité, affirme que les chefs ouvriers Francq et Verville ne détiennent pas les compétences nécessaires pour intervenir sur la question scolaire: "Voilà de pauvres ouvriers qui sont exposés à être mal renseignés sur l'importante question scolaire. Qu'attend-on pour arracher notre classe ouvrière à ces meneurs mal inspirés ${ }^{54}$ ?" Trois semaines plus tard, le journal revient à la charge, comparant Francq à l'encyclopédiste Diderot et au dirigeant socialiste français Jean Jaurès pour leurs opinions anticléricales, avant de conclure que les chefs ouvriers - Francq, Verville et Arcand - «sont imbus des doctrines les plus malsaines et bien près d'être en communauté d'idées et d'aspirations avec les révolutionnaires et les anticléricaux du vieux monde ${ }^{55}$ ».

Pour sa part, Langlois réclame dès décembre 1905 la création d'une commission d'enquête sur l'opportunité de fusionner les commissions scolaires montréalaises. En mars 1906, il revient à la charge à l'Assemblée législative réclamant cette fois plus d'intervention de la part de l'État dans la gestion des écoles primaires. Satisfait des interventions de Langlois à Québec, le CMTM lui écrit pour le féliciter et l'inviter à continuer son action en faveur de l'éducation et aussi de la municipalisation des services publics ${ }^{56}$. En mars 1908, Langlois soumet un projet de loi en faveur de l'uniformité des livres pour l'ensemble de la province. Le projet de loi sera 
battu, mais Langlois le représentera lors de la session de 1911. Toujours en 1908, il mène aussi une campagne à Québec contre son propre gouvernement qu'il rend directement responsable des trop faibles salaires des institutrices ${ }^{57}$. Ainsi, lui aussi doit subir les foudres de Jules Dorion de L'Action sociale qui n'hésite pas à présenter Langlois comme un homme "dangereux » dont les actions et les réformes, qui « ressemblent trop à ceux de certains politiciens d'outre-mer», ont "une forte saveur de radicalisme ${ }^{58} "$.

À la suite des pressions constantes des libéraux radicaux et des syndicalistes, et malgré l'opposition du clergé montréalais, le premier ministre Gouin décide de nommer une commission royale d'enquête qui portera uniquement sur les écoles catholiques de Montréal. Créée en juillet 1909, la Commission sera dirigée par le sénateur Raoul Dandurand et aura pour mandat de traiter uniquement de la question de la centralisation des commissions scolaires en une commission unique et de la question de l'élection des commissaires d'écoles. Le projet de centralisation sera défendu par les groupes ou personnes suivants : les porte-parole de la classe ouvrière comprenant le CMTM, le Parti ouvrier, la Ligue de l'instruction publique et Albert Saint-Martin, le militant socialiste qui témoignera en son nom personnel - le notaire Victor Morin, représentant l'Association immobilière de Montréal, et le juge Eugène Lafontaine, commissaire de la Commission des écoles catholiques de Montréal (CÉCM) de 1907 à 1909 puis président de 1919 à 1928.

Très ambitieux, le mémoire du Parti ouvrier présenté devant les commissaires ne se limite pas aux fusions des commissions scolaires, mais il réclame également l'instruction gratuite et obligatoire, le relèvement des salaires des institutrices, l'uniformité des manuels scolaires et la mise sur pied d'un ministère de l'Instruction publique. Fait à souligner, cette 
dernière revendication ne sera satisfaite qu'au moment de la création du ministère de l'Éducation en 1964. Les dirigeants ouvriers justifient ainsi leurs revendications :

Pour nous, cette question de réformes scolaires n'est pas une question religieuse, mais uniquement nationale. [...] Tout observateur impartial est forcé de reconnaître que la population canadienne-française, malgré ses talents naturels, son énergie au travail, son ambition et ses efforts de tous genres, est reléguée au second et troisième plan dans la Confédération.

Dans la haute finance, dans la grande industrie, dans les grandes administrations de chemin de fer et de navigation, dans les grandes compagnies de services publics, telles que compagnies d'éclairage, compagnies de tramways, etc., le Canadien-français n'existe virtuellement point. Nous trouvons les nôtres dans les petits magasins, dans les métiers durs et pénibles, dans les situations les moins élevées et partout où les salaires sont dérisoires. [...]

L'école, telle qu'elle existe actuellement, est une cause de faiblesse pour la province de Québec. L'école, telle qu'elle devrait exister, serait un instrument de relèvement et de force entre les mains des Canadiens-français ${ }^{59}$.

Le rapport final de la Commission déposé en 1911 favorise la centralisation des trente et une commissions scolaires, ce qui restera lettre morte dans l'immédiat. Durant les années subséquentes, plusieurs commissions scolaires, ne pouvant supporter de lourds déficits financiers, n'auront d'autre choix que de s'annexer à la CÉCM. À la suite d'une entente entre Lomer Gouin et $\mathrm{M}^{\mathrm{gr}}$ Bruchési, la fusion des commissions scolaires au sein de la CÉCM sera finalement adoptée le 22 décembre 1916 et appliquée à partir du $1^{\text {er }}$ juillet 1917. L'uniformité des livres scolaires sera assurée par le Bureau central chargé 
de l'administration financière de la CÉCM. Ainsi, les ouvriers avaient pleinement raison de croire que la centralisation faciliterait l'obtention de l'uniformité des livres, une de leurs principales revendications.

Lors de la session de novembre 1912 - considérée comme la session de l'éducation - le docteur John Finnie dépose, pour une deuxième année consécutive, un projet de loi en faveur de l'instruction obligatoire pour les écoles protestantes qui prévoit aussi la gratuité des livres et la gratuité scolaire. Appuyé et défendu par Langlois qui aurait tout de même préféré "voir un bill d'obligation pour tout le monde", le député de Saint-Louis défend le projet de loi Finnie sur le principe de l'égalité des droits et rappelle aux membres de l'Assemblée législative que les dirigeants ouvriers - et il les nomme : Verville, Ainey, Giroux, Arcand, Francq — sont en faveur de ce projet depuis longtemps : «Ils viennent tous les ans, depuis une dizaine d'années, faire un pèlerinage auprès du gouvernement pour réclamer cette réforme ${ }^{60} . »$

Lucides malgré la défaite du projet de loi qui sera battu par une majorité de 56 voix (62 contre 6), les dirigeants syndicaux ne peuvent s'expliquer ce résultat que par l'omniprésence et la crainte que suscite l'Église catholique auprès des pouvoirs publics: "We are under the impression that individually all the ministers - or at least the majority of the ministers - are in favor of this measure but as it has been made a religious question in our province, all goes to show that they wont dare tackle it for a long time to come ${ }^{61}$.» Langlois semble du même avis puisqu'il est convaincu qu'un scrutin secret donnerait une majorité en faveur de ce projet de loi.

\section{L'élection municipale montréalaise de février 1908}

$\mathrm{Si}$ la question importante de la réforme scolaire demeure la pierre angulaire, le ciment, de l'alliance entre les syndicats 
internationaux et les libéraux radicaux, il n'en demeure pas moins que les deux parties présentent plusieurs autres réclamations similaires. Sur la scène municipale, ils feront ensemble la lutte contre les trusts et les monopoles et réclameront une plus large implication de l'État dans la réglementation des services publics par la municipalisation de certains services comme ceux du gaz, de l'électricité et des tramways. L'importante question de l'abolition de la qualification foncière et le problème de patronage et de corruption à l'Hôtel-de-ville de Montréal fait également partie des causes communes que défendront les syndicalistes internationaux et les libéraux radicaux.

Cette situation se manifestera par exemple lors de l'élection municipale de février 1908 qui oppose à la mairie Louis Payette, le candidat de l'establishment du Parti libéral, au candidat des libéraux radicaux et des syndicalistes internationaux, Philippe Roy, membre fondateur du premier Parti national d'Honoré Mercier en 1872, député libéral provincial de Saint-Jean depuis 1900 et président de l'Assemblée législative. Si Roy présente une feuille de route impressionnante au niveau provincial, son manque d'expérience des affaires municipales constitue une faiblesse majeure face à un homme aussi expérimenté que Louis Payette qui représente le quartier Saint-Louis depuis 1902 et qui agissait en 1907 à titre de président du comité des finances au conseil municipal. De plus, Payette profite d'appuis majeurs d'une partie de la bourgeoisie montréalaise puisqu'il est le digne représentant du trust de la Montreal Light Heat and Power Company (MLHPC) au conseil municipal ${ }^{62}$. La Presse appuie Payette, mais Le Canada, le journal officiel du Parti libéral dirigé par Langlois, prend position en faveur de Roy, malgré l'opinion de l'establishment libéral — principalement Wilfrid Laurier et L.-O. David — qui préfère la candidature de Payette. 
Lors d'une assemblée politique organisée en faveur de Roy au Monument national le 21 janvier 1908, Francq, Ainey et G.-R. Brunet sont présents pour l'appuyer. Francq, qui prend la parole à titre de secrétaire général du Parti ouvrier, mentionne que le Parti a décidé de combattre la candidature de Louis Payette, le protecteur des monopoles. Pour Francq, Payette est le "grand fauteur de la mauvaise administration à l'hôtel de ville [sic] $]^{63}$ ", tandis que Roy se présente comme le défenseur de la classe ouvrière.

Le programme politique de Roy a de quoi plaire aux dirigeants ouvriers puisqu'il s'oppose ouvertement au monopole de la MLHPC et il favorise l'abolition de la qualification foncière pour les candidats au poste de maire et de conseillers ${ }^{64}$. D'ailleurs, tant le CMTM que le Parti ouvrier, ont déjà décidé qu'ils n'appuieraient que les candidats qui s'engageront à travailler en fonction de l'abolition de la qualification foncière, tout en précisant que ces candidats ne devront pas être issus du groupe des vingt et un échevins qui ont voté en faveur du renouvellement du contrat avec la MLHPC orchestré par Payette en $1905^{65}$. Dans Le Canada, Langlois justifie sa décision d'appuyer la candidature de Roy de la même façon: "Aujourd'hui comme hier, nous ne pouvons approuver les hommes qui ont formé bloc (les 21) pour favoriser un monopole odieux ${ }^{66}$.»

Comme le principe de la qualification foncière, qui oblige les citoyens qui veulent se porter candidats à l'échevinage à posséder une propriété immobilière d'une valeur d'au moins deux mille dollars, empêche les ouvriers de se porter candidats, le Parti ouvrier doit se contenter d'endosser la candidature des «bourgeois » qui promettent de suivre son programme. À cette élection, en plus de la candidature de Roy, le Parti ouvrier endossera officiellement sept autres candidats. Il faut dire que ces candidats n'ont pas à appuyer l'ensemble 
du programme ouvrier, mais seulement l'abolition de la qualification foncière. Dans une lettre qu'il fait parvenir à Francq, Roy n'hésite pas à affirmer qu'il adoptera le programme du Parti ouvrier, "qui se résume à une simple clause : abolition de la qualification foncière pour remplir les fonctions de maire et d'échevin dans cette Cité ${ }^{67}$ ". L'urgence de cette mesure semble dicter les choix des dirigeants du Parti ouvrier.

Outre Philippe Roy, d'autres candidats comme Georges Vandelac et Léandre Ouimet auront droit à un appui soutenu de la part des dirigeants du Parti ouvrier. Les chefs ouvriers - Joseph Ainey, G.-R. Brunet, Isidore Tremblay, L.A. Girard, Octave Jetté, Achille Latreille, Joseph Larocque, Érasme Charbonneau, etc. - assistent en grand nombre aux assemblées politiques de Vandelac et plusieurs d'entre eux prononcent des discours en sa faveur. Latreille et Ainey s'en prennent particulièrement à son opposant, W.-J. Proulx, qu'ils accusent de trahison envers la classe ouvrière puisqu'il a voté en faveur du renouvellement du contrat de la MLHPC, même après avoir obtenu l'appui du Parti ouvrier lors des élections précédentes $^{68}$. Dans un discours prononcé en présence d'une forte délégation de dirigeants ouvriers, Vandelac est fier d'affirmer qu'il a endossé avec plaisir le programme des ouvriers ainsi que celui des hommes d'affaires!

En somme, les résultats de cette élection seront plutôt décevants pour les libéraux radicaux et pour les syndicalistes puisque Roy sera battu par Payette à la mairie à raison de 14 710 voix contre 11 914. Seulement deux des sept candidats appuyés par le Parti ouvrier seront élus - J.-P. Gadbois et Emery Lespérance - tandis que quatre d'entre eux, dont Georges Vandelac et Léandre Ouimet, seront battus. Le septième candidat, J.-A. Séguin, dans Saint-Gabriel, se serait désisté avant la mise en candidature officielle ${ }^{69}$. Par contre, les pressions continues des syndicalistes internationaux contri- 
bueront finalement à l'abolition de la qualification foncière à Montréal quelques années plus tard, en 1912.

Pour les syndicalistes internationaux, les avantages liés à ces alliances sont fort nombreux. Celles-ci leur permettent, tout d'abord, d'obtenir une voix à l'Assemblée législative prête à défendre leurs intérêts et à rappeler périodiquement au gouvernement la présence des organisations syndicales au Québec. À cet égard, l'intervention de Godfroy Langlois lors du débat sur le projet de loi Finnie constitue un bon exemple. Il faut rappeler qu'à cette époque, à Montréal, le Parti ouvrier ne fait élire aucun candidat lors d'élections provinciales, subissant des défaites successives en 1904 (Alphonse Verville et Achille Latreille), en 1908 (Gustave Francq) et en 1912 (G.-R. Brunet et Narcisse Arcand). À Québec, Joseph-Alphonse Langlois se fait élire en 1909 sous la bannière ouvrière, mais il passe rapidement sous l'emprise des libéraux modérés. Dans une certaine mesure, les syndicalistes se fient aux libéraux radicaux pour les représenter à Québec. De plus, en obtenant l'appui de certains membres du gouvernement, les organisations syndicales atteignent indirectement un de leurs objectifs majeurs, soit l'obtention d'une reconnaissance officielle de la part des pouvoirs publics. Les dirigeants internationaux veulent avoir leur mot à dire dans les affaires publiques et être considérés au même titre que d'autres groupes de pression comme la Chambre de commerce de Montréal ou le Board of Trade. On rejoint ainsi la notion de juste représentation dans les instances gouvernementales qui est inscrite dans la constitution du Parti ouvrier depuis 1904 et que les travaillistes défendent avec ardeur. Il faut aussi souligner le fait que les journaux de Langlois, principalement Le Pays, donnent de la visibilité aux syndicalistes internationaux qui n'ont pas de véritable journal avant la création du Monde ouvrier en 1916. De fait, entre 1910 et 1914, année du départ de Lan- 
glois pour la Belgique, nous avons recensé plus d'une centaine d'articles touchant les questions ouvrières dans Le Pays. Le journal défend constamment les positions des syndicats internationaux.

\section{$* * *$}

Lorsqu'on s'arrête aux combats et aux revendications soutenues conjointement par les syndicalistes internationaux et les libéraux radicaux depuis le début du siècle, on doit reconnaître l'influence que le radicalisme français a exercée sur ces organisations et sur une partie de la société québécoise de cette époque. Même s'il est vrai que les syndicalistes internationaux sont d'abord et avant tout influencés par le syndicalisme de métier américain, représenté ici par la Fédération américaine du Travail de Samuel Gompers, il n'en demeure pas moins qu'il faut aussi reconnaitre l'influence du radicalisme français. Outre le fait que les principales revendications des syndicalistes et des libéraux soient similaires à celles des radicaux français, nous savons que des dirigeants comme Gustave Francq et Godfroy Langlois - entre autres - connaissaient la politique européenne et française et avaient un penchant pour le mouvement radical. Des hommes comme Francq et Langlois sont au fait des questions internationales. À la lecture des éditoriaux de Francq publiés dans Le Monde ouvrier, on s'aperçoit qu'il est perméable à plusieurs courants de pensée. Belge d'origine, il est influencé depuis sa jeunesse par la situation belge (Parti ouvrier, Ligue démocratique chrétienne, mouvement coopératif, etc.), mais également par le contexte français (Troisième République et radicalisme), par le travaillisme anglais et par le syndicalisme américain (Fédération américaine du travail).

$\mathrm{Au}$ Québec, l'influence du radicalisme français se fait aussi sentir dans d'autres organisations comme la franc-ma- 
çonnerie et la Ligue de l'Enseignement. En 1969, dans La question du Québec, le sociologue Marcel Rioux, développant la thèse du monolithisme idéologique, dépeignait la période se terminant en 1960 comme " un long hiver qui a duré plus d'un siècle ${ }^{70} »$. Or, malgré la très grande influence de l'Église catholique, nous savons aujourd'hui que le pluralisme idéologique existait au Québec au tournant du siècle et que le radicalisme constituait une des composantes de cette pluralité.

\section{NOTES}

${ }^{1}$ L'auteur tient à remercier les évaluateurs anonymes et le comité de direction de Mens pour leurs commentaires pertinents.

${ }^{2}$ Pierre Elliott Trudeau, dir., La grève de l'amiante, Montréal, Cité Libre, 1956, 430 p. ; Fernand Ouellet, "La Révolution tranquille, tournant révolutionnaire ?", dans Thomas S. Axworthy et Pierre Elliott Trudeau, dirs., Les années Trudeau. La recherche d'une société juste, Montréal, Le Jour éditeur, 1990, pp. 333-362 ; Marcel Rioux, La question du Québec, Paris, Seghers, 1969, 184 p. ; Hubert Guindon, «La modernisation du Québec et la légitimité de l'État canadien ", Recherches sociographiques, vol. 18, $\mathrm{n}^{\circ} 3$ (1977), pp. 337-366 ; Kenneth McRoberts et Dale Postgate, Quebec: Social Change and Political Crisis, Toronto, McClelland and Stewart, 1976, $216 \mathrm{p}$.

${ }^{3} \mathrm{Au}$ sujet de la présence du libéralisme au Québec au début du $\mathrm{XX}$ e siècle, on consultera : Yvan Lamonde, dir., Combats libéraux au tournant $d u X X^{e}$ siècle, Montréal, Fides, 1995, 285 p.

${ }^{4}$ Roger Le Moine, Deux loges montréalaises du Grand-Orient de France, Ottawa, Presses de l'Université d'Ottawa, 1991, 188 p. ; Patrice Dutil, L'Avocat du diable. Godfroy Langlois et le libéralisme progressiste dans le Québec de Wilfrid Laurier, Montréal, Éditions Robert Davies, 1995, 286 p. ; Éric Leroux, Gustave Francq. Figure marquante du syndicalisme et précurseur de la FTQ, Montréal, VLB éditeur, 2001, $371 \mathrm{p}$.

${ }^{5}$ Gérard Baal, Le Parti radical de 1901 à 1914, Thèse de doctorat d'État (histoire), Université de Paris I, 1991, p. II.

"Serge Berstein, Histoire du Parti radical, Paris, Presses de la Fondation nationale des sciences politiques, 1980, p. 25. 
${ }^{7}$ Guy Bélanger, «La première caisse populaire de Montréal fut fondée par des leaders du mouvement ouvrier ", La Revue Desjardins, vol. 3 (1994), pp. $22-23$; Leroux, Gustave Francq, pp. 82-85.

${ }^{8}$ Bernard Dionne, Le syndicalisme au Québec, Montréal, Boréal Express, 1991, p. 21 ; Jacques Rouillard, Histoire du syndicalisme québécois, Montréal, Boréal, 1989, p. 77 (tableau 2.2).

${ }^{9}$ Bernard Dansereau, Le mouvement ouvrier montréalais, 1918-1929 : structure et conjoncture, Thèse de doctorat (histoire), Université de Montréal, 2000, p. 131.

${ }^{10}$ Sur le Parti ouvrier, on consultera : Jacques Rouillard, «L'action politique ouvrière au début du $\mathrm{XX}^{\mathrm{e}}$ siècle ", dans Fernand Harvey, dir., Le mouvement ouvrier au Québec, Montréal, Boréal Express, 1980, pp. 115-214 ; Craig Heron, "Labourism and the Canadian Working Class », Labour / Le Travail, $\mathrm{n}^{\circ} 13$ (printemps 1984), pp. 45-76 ; Leroux, Gustave Francq, pp. 100-115.

${ }^{11}$ Éric Leroux, "Verville, Alphonse », Dictionnaire biographique du Canada, Volume XV (1921-1930), Sainte-Foy, Presses de l'Université Laval, 2005, pp. $1149-1152$.

12 Jean-Thomas Nordmann, Histoire des radicaux 1820-1973, Paris, La Table Ronde, 1974, pp. 16-24 ; Gérard Baal, Histoire du radicalisme, Paris, Éditions La Découverte, 1994, pp. 4-23.

${ }^{13}$ Le Monde ouvrier, 16 juillet 1927, p. 1.

${ }^{14}$ Ibid., 24 février 1923, p. 1.

${ }^{15}$ Albert Métin, Délégation ouvrière française aux États-Unis et au Canada, Paris, Édouard Cornély et compagnie éditeur, 1907, p. 61.

${ }^{16}$ Claude Nicolet, Le radicalisme, Paris, Les Presses Universitaires de France, 1967, pp. 43-48 ; Nordmann, Histoire des radicaux, p.125.

${ }^{17}$ Au sujet des revendications des syndicalistes internationaux, voir : Rouillard, Histoire du syndicalisme québécois, 535 p. ; Sylvie Murray et Élyse Tremblay, Cent ans de solidarité : bistoire du CTM (1886-1986), Montréal, VLB éditeur, 1987, 150 p. ; Bernard Dionne, Les Unions internationales et le Conseil des métiers et du travail de Montréal, Thèse de doctorat (histoire), Université du Québec à Montréal, 1988,834 p.

${ }^{18}$ Nicolet, Le radicalisme, p. 47.

${ }^{19}$ Au début du siècle, à Montréal, les syndicalistes internationaux, majoritaires et occupant les postes de direction, dirigent le Parti ouvrier et décident de l'orientation à lui donner. Malgré quelques soubresauts, cette hégémonie 
perdurera jusqu'au début de la Première Guerre mondiale (Leroux, Gustave Francq, pp. 93-136).

${ }^{20}$ La Patrie, 22 novembre 1906, p. 3.

${ }^{21}$ Dutil, L'Avocat du diable, p. 192.

${ }^{22}$ Ruby Heap, «La Ligue de l'Enseignement (1902-1904) : héritage du passé et nouveaux défis ", Revue d'bistoire de l'Amérique française, vol. 36, $\mathrm{n}^{\circ} 3$ (1982), pp. 339-373.

${ }^{23}$ Gustave Francq est lié de très près au journal Le Pays. Imprimé sur les presses de son imprimerie, la Mercantile Printing, depuis ses débuts en 1910, le journal est dirigé par Francq en 1920 et 1921. Il y aurait même écrit plusieurs éditoriaux sous divers pseudonymes au cours des années 1910 et 1920. En 1913, malgré la condamnation du journal par $\mathrm{M}^{\mathrm{gr}}$ Bruchési, il continue d'être imprimé par la Mercantile Printing qui y fait paraitre plusieurs publicités pour vanter les mérites de son atelier. Outre Le Pays, Francq entretient aussi des liens avec d'autres journaux radicaux, comme La vie artistique (1905) et $\mathrm{La}$ Lumière (1912), deux journaux imprimés sur ses presses. Dirigé par A. Morin, le mensuel La Lumière s'inscrit dans la lignée du rationalisme et du radicalisme français avec la présentation de textes de Diderot, Paul Sabatier, Anatole France, Georges Clémenceau, Victor Hugo et plusieurs autres. Ainsi, lorsque $L a L u-$ mière subit les foudres de $\mathrm{M}^{\mathrm{gr}}$ Bruchési, qui le condamne le 9 juin 1912, la Mercantile demeure l'imprimeur attitré du journal.

${ }^{24}$ Baal, Histoire du radicalisme, 1994, p. 14.

${ }^{25}$ Paul Nadon, La Franc-Magonnerie, Paris, Les Presses universitaires de France, 1982, pp. 22-23.

${ }^{26}$ Le Moine, Deux loges montréalaises, p. 23.

${ }^{27}$ Le Desoir, 2 août 1910, p. 1.

${ }^{28}$ Le Moine, Deux loges montréalaises, p. 66.

${ }^{29}$ Le Monde ouvrier, 19 mai 1923, p. 1.

${ }^{30}$ Le Monde ouvrier, 28 août 1920, p. 1.

${ }^{31}$ Voir à ce sujet: Dansereau, Le mouvement ouvrier montréalais, 19181929 ; Geoffrey Ewen, The International Unions and the Workers' Revolt in Quebec, 1914-1925, Thèse de doctorat (histoire), York University, 1998, 502 p.

${ }^{32}$ Andrée Lévesque, «Éva Circé-Côté (1871-1949) », dans Maryse Darsigny, dir., Ces femmes qui ont bâti Montréal, Montréal, Éditions du Remue-ménage, 
1994, p. 149 ; Lemieux, La loge L'Émancipation, pp. 8-12 ; Le Moine, Deux loges montréalaises, pp. 53 et 61.

${ }^{33}$ Andrée Lévesque, «Journaliste au masculin : Éva Circé-Côté (1871-1949) ", dans Évelyne Tardy, dir., Les Bâtisseuses de la Cité, Montréal, ACFAS, 1993, p. 88 .

${ }^{34}$ Le Pays, 9 avril 1910, p. 4.

${ }^{35}$ Dutil, L'Avocat du diable, p. 113.

${ }^{36}$ Ibid., pp. 112-113.

${ }^{37}$ Ibid., p.164. T.-D. Bouchard fait la même analyse que Dutil dans ses mémoires (T.-D. Bouchard, Mémoires, tome 2, Gravissant la colline, Montréal, Éditions Beauchemin, 1960, p. 96).

${ }^{38}$ Dutil, L'Avocat du diable, pp.164-165.

${ }^{39}$ La Presse, 10 novembre 1904, p. 1 ; Le Canada, 12 novembre 1904, p. 6.

${ }^{40}$ Robert Gagnon, Histoire de la Commission des écoles catholiques de Montréal, Montréal, Boréal, 1996, p. 92.

${ }^{41}$ La Vérité, 4 mars 1882.

${ }^{42}$ Souvenirs de la Fête du travail (illustré), 1900.

${ }^{43}$ Annette Hayward, «La littérature de la modernité et le libéralisme nationaliste au Québec entre 1899 et 1916 », dans Yvan Lamonde, dir., Combats libéraux au tournant $d u X X^{e}$ siècle, Montréal, Fides, 1995, pp. 159-184 ; Fernande Roy, «Le journal L'Autorité dans le cadre de la presse libérale montréalaise », dans Lamonde, dir., Combats libéraux au tournant du XX siècle, pp. 231-246.

${ }^{44}$ Ruby Heap, "Libéralisme et éducation au Québec à la fin du XIX ${ }^{\mathrm{c}}$ et au début du XX $\mathrm{XX}^{\mathrm{e}}$ siècles ", dans Yvan Lamonde, dir., Combats libéraux au tournant $d u X X^{e}$ siècle, pp. 99-118.

${ }^{45}$ Éric Leroux, « Les syndicats internationaux et la Commission royale d'enquête sur l'éducation de 1909-1910", Bulletin du RCHTQ, vol. 23, nº 1 (1997), pp. 5-14 ; Céline Bastien, Les syndicats internationaux et les réformes scolaires au Québec (1900-1930), Thèse de maitrise (histoire), Université de Montréal, 1997, 110 p. ; Thérèse Hamel, «L'obligation scolaire au Québec : enjeu pour le mouvement syndical et agricole ", Labour / Le Travail, no 17 (1986), pp. 83102.

${ }^{46}$ Robert Rumilly, Histoire de la province de Québec, tome XII, Les écoles du NordOuest, Montréal, Valiquette, 1944, pp. 101-102. 
${ }^{47}$ Dutil, L'Avocat du diable, pp. 47-50.

${ }^{48}$ L'Écho des Deux-Montagnes, 27 novembre 1890.

${ }^{49}$ Le Pays, 24 février 1912, p. 1.

${ }^{50}$ Robert Rumilly, Histoire de Montréal, tome 3, Montréal, Fides, 1972, p. 381.

${ }^{51} \mathrm{La}$ liste des livres français approuvés par les écoles catholiques en 1904 est un exemple éloquent de cette situation. On y dénombre en effet vingt-sept choix différents de livres de lecture, neuf choix de grammaire, quinze livres d'histoire dont près de la moitié date de 1860 , neuf choix de livres de géographie et treize choix de livres de mathématique (Liste des livres approuvés par les écoles catboliques par le Comité catholique du conseil de linstruction publique jusqu'au $1^{\text {er }}$ juillet 1904, Québec, 1904, 18 p.).

${ }^{52}$ Proceedings of The Trades and Labor Congress of Canada (TLCC), 1909, p. 23.

${ }^{53}$ L'Action sociale, 25 septembre 1909, p. 4.

${ }^{54}$ La Vérité, 13 novembre 1909, p. 140.

${ }^{55}$ La Vérité, 4 décembre 1909, p. 166.

${ }^{56}$ Procès-verbaux du Conseil des métiers et du travail de Montréal (CMTM), assemblée du 8 mars 1906, p. 25.

${ }^{57}$ Gagnon, Histoire de la Commission des écoles, p. 96.

${ }^{58}$ L'Action sociale, 30 mai 1908 , p. 4.

${ }^{59}$ Archives de la CÉCM, Commission royale d'enquête 1909-1910. Séance $d u$ 21 décembre 1909, mémoire du Parti ouvrier, pp. 16-18.

${ }^{60}$ Québec, Débats de l'Assemblée législative, $13^{\mathrm{e}}$ Législature, $1^{\mathrm{e} r e}$ session, séance du 27 novembre 1912, p. 182.

${ }^{61}$ Proceedings of TLCC, 1912, p. 37.

${ }^{62}$ Claude V. Marsolais, Histoire des maires de Montréal, Montréal, VLB éditeur, 1993, p. 198.

${ }^{63}$ La Patrie, 22 janvier 1908, p. 7.

${ }^{64}$ Le Canada, 22 janvier 1908, p. 10.

${ }^{65}$ La Presse, 6 décembre 1907, p. 13 ; 14 décembre 1907, p. 25.

${ }^{66}$ Le Canada, 29 janvier 1908, p. 5.

${ }^{67}$ Le Canada, 18 janvier 1908, p. 13. 
204

Mens

${ }^{68}$ Le Canada, 8 janvier 1908, p. 9.

${ }^{69}$ La Patrie, 20 janvier 1908, p. 3.

${ }^{70}$ Rioux, La question du Québec, p. 104. 